\title{
Perceptions relating to body size, weight loss and weight-loss interventions in black South African women: a qualitative study
}

\author{
Catherine E Draper ${ }^{1}$, Kesiah J Davidowitz ${ }^{1}$ and Julia H Goedecke ${ }^{1,2, *}$ \\ 'Division of Exercise Science and Sports Medicine, Department of Human Biology, Faculty of Health Sciences, \\ University of Cape Town, Cape Town, South Africa: ${ }^{2}$ Non-Communicable Disease Research Unit, South African \\ Medical Research Council, Francie van Zijl Drive, Tygerberg 19070, Parow, South Africa
}

Submitted 21 November 2014: Final revision received 27 March 2015: Accepted 15 April 2015: First published online 26 May 2015

\begin{abstract}
Objective: A higher tolerance for a larger body size has been associated with obesity in black South African (SA) women. The aim of the present study was to explore perceptions regarding body size and weight loss in a sample of black women from a low-income community in Cape Town, SA.

Design: Qualitative pilot study including five focus groups. Data were analysed using thematic analysis.

Setting: Khayelitsha, Cape Town, SA.

Subjects: Twenty-one black SA women.

Results: The majority of participants had positive perceptions of overweight/ obesity, which were influenced by community and cultural perceptions, but some inconsistencies were observed as overweight/obesity was also associated with ill health. Participants identified many benefits to weight loss, but due to the association with sickness, they were concerned about being stigmatised in their community. Although participants had knowledge about healthy eating, the main barrier to eating healthily included the perceived higher cost of healthier food and food insecurity. All participants saw exercise as a strategy to lose weight and improve health, and were interested in participating in a community-based exercise intervention, but negative community perceptions and conflicting views regarding who should lead the intervention were identified as barriers.

Conclusions: These findings highlight the complexities surrounding participants' perceptions regarding body size, weight loss and weight-loss interventions, and emphasise low socio-economic status as a barrier to change. The study also highlights the strong influence of cultural ideals and community perceptions on personal perceptions. These findings underscore the necessity for culturally appropriate weight-loss interventions in low-income, transitioning communities.
\end{abstract}

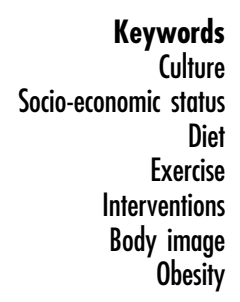

The prevalence of overweight and obesity is a global problem $^{(1)}$ and seems to be specifically evident in countries undergoing epidemiological transition, such as South Africa $(\mathrm{SA})^{(2,3)}$, with adult women being the most susceptible individuals ${ }^{(4)}$. Obesity is associated with an increased risk of non-communicable diseases and in 2000, excess body weight was ranked as the fifth highest risk factor for deaths in the SA population, with twice the number of deaths occurring in women compared with men ${ }^{(5)}$. The prevalence of obesity in SA is increasing, particularly in black SA women, in whom the prevalence has increased from $27 \%$ in 2003 to $39 \cdot 2 \%$ in $2012^{(6)}$.

Body image has been found to be an important factor associated with the higher prevalence of obesity in women, particularly in black women ${ }^{(7)}$. Body image has been defined as one's perceptions of one's body and body image discrepancy is the difference in perceived actual body image and perceived desired (ideal) body image ${ }^{(8)}$. Differences in body image are determined by the way women view their bodies (body image perception) and how they feel about their bodies (body image attitude) ${ }^{(9)}$. In SA women, body image is partly driven by culture ${ }^{(10)}$, urbanisation and socio-economic status ${ }^{(11,12)}$. Culture may be defined as the unique mutual beliefs, values and practices in a group, and can influence the behaviours of individuals by affecting their feelings, thoughts, acceptance and agreement of messages regarding health education $^{(13)}$. Within a relatively homogeneous community, it is possible that beliefs, values and practices are not consistent. However, in the context of the present study, 
we understand culture to refer to the beliefs, values and practices that could be considered the most common or dominant in a particular community.

Research has shown that black women have a higher tolerance for an increased body size compared with their white counterparts and show a preference for a larger body size ${ }^{(7,14,15)}$. Studies with black women in SA have found that obesity is positively associated with affluence, health, wealth, attractiveness, happiness and being treated well by one's husband ${ }^{(7,11,12,16)}$. Work conducted in SA has suggested that perceptions of weight are influenced by ethnicity, as only $27 \%$ of overweight and obese black women perceived themselves to be overweight compared with $100 \%$ of overweight and obese white women ${ }^{(4)}$.

Body image, tolerance for increased body size and cultural perceptions of body size are important factors to take into consideration when planning and implementing weight-loss interventions, which typically promote increased physical activity and healthy eating behaviours. However, many barriers to the adoption of these behaviours have been reported in lower-income settings, including China, Brazil, India and Nigeria ${ }^{(17)}$. These include the limited availability of affordable healthy foods in combination with the ready availability of high-fat foods. Similarly, the decline in physical activity has been attributed to an increase in sedentary employment, limited outdoor space ${ }^{(18)}$ and increased rates of street violence ${ }^{(19)}$. Furthermore, weight loss in some African cultures is associated with illness, most commonly HIV/AIDS and tuberculosis $(\mathrm{TB})^{(12,20)}$.

While many research studies have evaluated perceptions of body size and body image ideals in different settings, little research has been undertaken examining black SA women's perceptions regarding weight loss and the impact of culture on these perceptions. Therefore, the aim of the present study was to explore perceptions of body size, weight loss and weight-loss interventions in a sample of black women from a low-income community in Cape Town, SA. The findings of the research could be used in the development of a culturally appropriate weight-loss intervention.

\section{Methods}

\section{Research design}

The present study was a qualitative pilot study to investigate the issues relating to perceptions of body size, weight loss and weight-loss interventions. Considering the exploratory and descriptive nature of the study, a qualitative approach was deemed to be most suitable ${ }^{(21)}$. Semi-structured focus groups were believed to be an appropriate method to capture group perceptions due to their naturalistic quality, in that they can encourage discussions that resemble the everyday manner in which opinions are formed, conveyed and shared ${ }^{(22)}$. Furthermore, focus groups also capitalise on human beings' nature as generally social beings, making focus group interactions enjoyable and therefore easier to conduct ${ }^{(23)}$. This characteristic of focus groups could be helpful in a setting such as the low-income one where the study was conducted, where participants may be unfamiliar with the nature of focus groups.

\section{Participants}

A convenience sample of black SA women ( $n$ 21, 24-51 years old) from a low-income community (Khayelitsha, in Cape Town) was used for the present study. Khayelitsha is a township with a high population density (9000 inhabitants $/ \mathrm{km}^{2} v \cdot 42 \cdot 4 / \mathrm{km}^{2}$ for SA), with a majority (99\%) black African population ${ }^{(24)}$. There are high rates of poverty and unemployment $(38.3 \%)$ and a substantial proportion of people (55\%) living in informal dwellings ${ }^{(24)}$. In 2010 , the prevalence of HIV among antenatal clinic attendees was $26.3 \%$ and 6000 case findings of TB were reported ${ }^{(25)}$.

The study was originally designed to recruit premenopausal women from the same age group (20-45 years) included in previous studies (by J.H.G.) examining obesity and metabolic risk in black SA women from Khayelitsha ${ }^{(26-28)}$. Specifically, in a 5-year longitudinal study of freeliving women from Khayelitsha, significant increases in body weight ( $8 \%$ increase) and metabolic risk were observed, which highlighted the need to explore suitable interventions to combat obesity in this population ${ }^{(28)}$.

Inclusion criteria for participants included being female, 20-55 years old (to allow for an increase in age since the previous studies) and resident in Khayelitsha. A fieldworker (resident in Khayelitsha), through local church groups and other community networks, recruited the participants for the study. The fieldworker contacted potential participants telephonically or in person, explained the nature of the study and asked them if they were willing to participate in a focus group, also if they knew of other women who would be willing to participate. The fieldworker then followed up individuals who indicated a willingness to participate. The study was conducted according to the guidelines laid down in the Declaration of Helsinki and all procedures involving human subjects were approved by the Human Research Ethics Committee of the Faculty of Health Sciences of the University of Cape Town (REC REF 101/2004, Version 5, March 2012). Written informed consent was obtained from all participants.

\section{Focus groups}

Five semi-structured focus groups were held, all 30-45 min in duration, with a range of two to ten women per group. The small numbers in some of the groups are an indication of the challenges often experienced in recruiting research participants for community-based research. These focus group sessions took place in a centrally located church in Khayelitsha. Light refreshments were provided at each 
focus group session; participants were not paid for their participation.

Since the home language of all participants was Xhosa, the fieldworker was present to translate where necessary. Focus group discussions were audio recorded and the facilitator took additional notes during each of the sessions. The audio recordings were translated and then transcribed verbatim in English.

At the start of the focus group discussion, participants were also asked to comment on a set of three photographs, which included a normal-weight, overweight and obese black woman. Thereafter, the discussion was facilitated with guide questions that were designed to stimulate discussion among participants. These included questions regarding their perceptions of overweight/obesity, an overweight/obese person, a thin person and weight-loss strategies, as well as questions regarding their own or others' experiences of weight loss and their suggestions for weight-loss interventions in their community, including diet and exercise strategies.

\section{Antbropometry}

Participants' BMI was calculated in order to contextualise the focus group findings. Participants' height and weight were taken at the end of the focus group sessions, so that their impressions of their measurements, or other participants' impressions of their measurements, would not influence their contributions to the discussion. The mean BMI of the participants was 32.0 (SD 8.0 ) $\mathrm{kg} / \mathrm{m}^{2}$, with $74 \%$ of participants being classified as overweight or obese.

\section{Analysis}

The focus group recordings were transcribed verbatim by a third party. These transcripts were read through initially to obtain a general sense of participants' responses. A coding framework of themes was developed based on the guide questions and also took into consideration any additional issues that were raised in the discussion of the photographs that participants were shown. The four main themes within this framework that were believed to best represent participants' responses were: (i) weight and body size perceptions; (ii) weight-loss perceptions; (iii) diet and diet interventions; and (iv) exercise and exercise interventions. Using the coding framework, transcripts were coded according to thematic content in order to identify 'repeated patterns of meaning, ${ }^{(29)}$. Once the transcripts had been coded, quotes for each code were grouped together and summarized, and specific quotes were chosen that best represented the themes within the coding framework.

In an attempt to enhance the validity of the data, all research participants were invited to a feedback session with the research team, which was held at the same local community venue as the focus groups. The purpose of the feedback session was to thank the participants for their contribution to the focus groups (they were given lunch and a few small gifts) and to present the research findings, allowing them an opportunity to respond. From the participants' responses, it was evident that the findings were an accurate reflection of their views.

\section{Results}

\section{Weight and body size perceptions}

Most participants felt that being overweight/obese was acceptable and desirable, and agreed that they liked to be 'fat'. Being 'fat' meant that they would not be judged by their peers or by the community, or that community members would not comment upon their weight status. They described that being overweight/obese revealed the female figure and that clothes such as dresses and skirts would be well suited to an overweight/obese figure. Having a 'figure' was important to the participants and they felt that this made them more attractive to men from their community (a 'figure' included being overweight/ obese with a larger buttocks and hips and a smaller upper body):

'Our culture says that we are supposed to be fat. You must have structure, you must be beautiful. In other words, our culture does not allow women to be thin. They say that someone who is fat is sexier.' (Focus group 1)

However, some participants disagreed and stated that they did not like to be overweight/obese, and that someone who is overweight/obese should lose weight. These participants felt that being overweight/obese was something to be ashamed of and that it would make the person feel uncomfortable, making it difficult for them to find clothing that fits. Furthermore, when shown the picture of an obese individual, all participants mentioned that the person portrayed in the picture was 'too fat', because of being stressed, eating unhealthy food or not participating in physical activity. All participants agreed that being overweight/obese was not necessarily a sign of wealth:

'Even if you have money you can be fat, even if you don't have money you can be fat.' (Focus group 1)

Participants acknowledged the risks to health associated with being overweight/obese, which included high blood pressure, diabetes, heart disease, stroke and sleep disorders. It was also mentioned that being overweight/obese would make it difficult for that individual to exercise:

'Overweight is not good because you got high blood [pressure], heart attack and so many diseases. Your heart will be covered in fat. It will be impossible for you to exercise, you won't last 5 minutes doing exercise.' (Focus group 5)

When shown the picture of a slender woman, most of the participants agreed that she was 'nice looking', healthy and sexy. However, there were some participants who felt 
that this woman was too thin, and that the community's perception of a slender woman would be negative, stating that she could have a disease, is unhealthy or could be using drugs. The most common diseases mentioned were HIV, TB and diabetes:

'We always think that the person is sick. It's obvious! That person has TB, HIV. We always assume even if we don't know what's going on with that person.'

(Focus group 4)

However, participants in one group mentioned that they are able to see the physical differences between someone who is ill, someone who is naturally thin and someone who chooses to be slender:

'... you can easily see a person who is sick or not sick. The person who is thin because she is sick, they will be white and their eyes will go inside. Their skin will be attached to their bones and their skin will be dry because they are dehydrated and the skin will be pale.' (Focus group 5)

'You will see this person is dehydrated and sick. Their walks would not be straight; you will see that this person is nearly to their death.' (Focus group 5)

\section{Weight-loss perceptions}

Some participants mentioned that people appear to be happier when they are thinner, and they are healthier once they have lost weight. Many benefits of weight loss were mentioned by the participants, including longevity and a decrease in risk factors for diseases such as hypertension, diabetes and CVD:

'If you lose weight and exercise you will have a longer life. You won't go to the doctor all the time. Normally the person would go to the doctor feeling ok and the doctor will tell you have high blood pressure even though you went to the doctor for the normal check-up routines.' (Focus group 5)

Participants were able to give examples of weight-loss strategies that someone might adopt in order to lose weight, and these included diet, exercise, weight-loss supplements (popular among all participants), stress and a weight-loss belt (those that vibrate when tied to a particular part of the body).

Many participants disagreed with the positive view of weight loss and explained that the community would stigmatise a woman attempting to lose weight, especially if she was older:

'She would not be happy [to lose weight] because in our community it would be perceived that there is something wrong with her. She would think that the community is looking at her. So she would feel insecure.' (Focus group 1)
'They would still judge her, because they say the person is sick and now wants to cover it up by dieting and exercise.' (Focus group 3)

Many of the participants had not attempted to lose weight, although some of the participants mentioned that they knew someone who was taking a weight-loss supplement and exercising in order to lose weight, but thought this individual was 'insane' for taking these measures. One participant said that when she was working she could go to the gym, as she could afford to do so, and instead of having meals she would have diet milkshakes in order to lose weight. A few other participants mentioned that losing weight, as with thinness, was associated with sickness, having no money or work, or stress.

\section{Diet and diet interventions}

In terms of participants' perceptions of dietary changes as part of a weight-loss intervention, most participants understood dieting to involve reducing their food intake, eating healthier foods and using less fat and oil in food preparation, all of which were positively associated with health:

'Diet means eat less fat, drink water, eat veggies, fruits, not too much sugar, less milk and low fat.' (Focus group 4)

However, negative community perceptions around dieting also exist, as mentioned by one participant:

'She is high class, because she is not eating what the rest of the community is eating.' (Focus group 1)

In terms of barriers to healthy eating in the community, the most common point raised by the participants was financial insecurity and the price difference between healthier foods and unhealthy or fatty foods. Many of them explained that they are unemployed and eat what is affordable. Most felt that healthy foods, such as vegetables and fruits, are more expensive than buying the cheaper foods frequently eaten in their community, including vetkoek (deep-fried dough), hot chips, derms (sheep intestines), sheep and pig's head:

'We have nothing. If there is melon in the house I eat, I eat and get full. At the end I am full.' (Focus group 5)

'The cucumber and veggies and stuff are expensive. All the food with fat are cheap, vetkoek, derms, pork.' (Focus group 4)

A few participants disagreed with this view and felt that fatty foods were more expensive than healthy foods. They explained that when they were unemployed the healthier food seemed more expensive, but when they were employed the healthier food seemed more affordable.

One of the staple foods of black South Africans is maize meal, or pap, which is relatively inexpensive, easy to access and can be consumed in large quantities at most meals of the day. A comment from one participant 
highlighted the extent to which this staple food may be a barrier to healthy eating. This comment also suggests that there may be a degree of emotional eating among women from this community, which may be a consequence of unemployment:

'They just limit their food intake and starchy food. You can't eat mieliepap in the morning and want to lose weight. The reason I don't get really thin is I eat stywe [stiff] pap, meals like samp and beans I don't like. The only thing that makes me full is pap. I eat anything that comes to my mouth. I eat too much because I'm not working.' (Focus group 5)

All participants said that they would be very interested in taking part in a dietary intervention where healthy foods were supplied to them:

'We are suffering because we don't have money; we are not employed to buy expensive food. If someone came and offered us the food, then we would take part.' (Focus group 5)

\section{Exercise and exercise interventions}

Although 'exercise' and 'physical activity' are not synonymous, exercise was used in the present study instead of physical activity since it is a more familiar term for participants. In addition, the terms 'physical activity' and 'exercise' would be the same when translated into Xhosa, so that the nuanced difference between these terms would be lost in translation. Furthermore, participants tended to refer to exercise as intentional physical activity that would be conducted in their leisure time.

All participants agreed that someone who is overweight should exercise to become healthier, and suggested activities such as walking, running, aerobics, push-ups, ball games, lifting weights or watching exercise classes on television. Participants also mentioned that the individuals should keep themselves constantly busy (e.g. by cleaning the house) and they should not spend too much time sleeping during the day. Many thought it would be a good idea to walk for transport in order to save money and increase their activity levels:

'Will go to the gym for example, she'll run, and even on TV she should watch the TV and do the exercises on the TV. Instead of using transport she should try to walk.' (Focus group 3)

However, participants explained that the people who exercise in the community are criticised, although individuals who exercise to keep fit for sports and younger people who exercise are not judged by the community. These participants mentioned that community members believe that an older person is wasting his/her time by exercising and losing weight:

'Some of them look at them like they are crazy. Some of them are fine; they don't look crazy because they want to be fit. They want to do exercise to be fit or for sport. But those who are looking think they are crazy sweating, running in the sun looking crazy while we are drinking our beer.' (Focus group 5)

'It's not important what they are doing because they look at the person and undermine them and think that this person is too old for the thing that he/she is doing.' (Focus group 3)

Other perceived barriers to exercising were the lack of facilities and equipment in their community and the lack of financial means to join an outside gym, since most participants were unemployed.

Many participants expressed interest in participating in an exercise intervention if there was space and equipment available to them, and felt that other community members would also be interested. Suggested activities included dance classes, boxing classes, playing ball games such as netball, and performing exercises like skipping, strengthening activities and cycling. Opinions differed regarding the preferred intensity of the exercise, with lower-intensity exercise being associated with fun and greater adherence by community members:

'They must enjoy exercise because if they overdo it then they will stop. It's possible for them to stop in the middle or drop the sessions.' (Focus group 3)

Those participants who felt that the community would not support an exercise intervention were also not willing to participate in such an intervention:

'There are people that will be interested. Things like dance. But not us, the only thing we focus on is drinking and to get drunk. Tell us about something like booze; don't tell us there is training at Wanda or Matshaya [local informal drinking establishment].' (Focus group 5)

There were conflicting views on who should lead community-based exercise sessions, should these be implemented. A few participants felt it would be better if someone from the community gave the classes, as they would feel at ease with someone they know and would be able to communicate better with one another:

'It should be someone we know. We want someone from the community so that we can understand each other and feel more comfortable.' (Focus group 1)

Others felt that an unknown person from outside the community should lead the exercise sessions, as they would be less judgemental, and they would therefore be more likely to listen to this person. They also mentioned that they would not trust someone from within their community, although some comments suggested that 
there was also mistrust of those from outside the community. Most of the participants felt that they would be most comfortable with both someone from within the community and someone from outside the community:

'We want someone who does not know us, so that person will not be talking about us to someone. We won't respect the person from the community. Someone from outside the community would be taken seriously. And this person would push us to work.' (Focus group 4)

'Why is someone from the community better? Even if a person from the community is going to facilitate the session, someone from outside the community giving the training should be present so that people from the community can see that this is happening and that those people are serious. The people feel misled. There were people who came to the community before and why give people hope and don't fulfil their promises, so the people want to see.' (Focus group 2)

\section{Discussion}

The findings of the present pilot study highlight a number of contrasting views and therefore the complexities surrounding the participants' perceptions regarding body size, weight loss and weight-loss interventions, and the extent to which low socio-economic status can be a barrier to change. The study also emphasises the strong influence of cultural ideals and community perceptions on personal perceptions. These findings underscore the necessity for culturally appropriate weight-loss interventions in communities such as Khayelitsha.

Khayelitsha may be characterised as a transitioning environment, with rapid urbanisation and economic development ${ }^{(30)}$. It is possible that during such a transition, a wide range of views can coexist on issues such as body size and weight loss. Participants' comments regarding body size are consistent with the findings of other SA studies ${ }^{(4,7)}$ which have suggested that black SA women prefer a larger body size. Similar findings are evident in other cultures such as Samoan populations of the Pacific Islands ${ }^{(31)}$, the women of Saudi Arabia ${ }^{(32)}$, women of Caribbean descent living in the $\mathrm{UK}^{(33)}$ and African-American women in the USA ${ }^{(34-36)}$.

The findings of previous studies suggest that many women of African descent view obesity not only as attractive but also as a sign of wealth and affluence $^{(7,12,16,20)}$. This view was not evident among participants in the present study, who felt that obesity did not necessarily represent wealth, but rather it could be a sign that someone is consuming unhealthy foods. These comments, along with comments about the health benefits of weight loss, indicate that a lack of knowledge may not necessarily be a barrier to weight loss in this group of women, although other studies have suggested that some ethnic and cultural differences in weight and weight management may be as a result of a lack of knowledge or education $^{(37,38)}$. Participants' knowledge, however, seemed to be overshadowed by community perceptions that weight loss is regarded as a sign of diseases such as HIV and/or TB, which has been found in other African settings $(7,12,16,20)$.

From the study findings it could be argued that in addition to poverty, community perceptions pose the greatest barrier to behaviour change associated with weight loss and could potentially present a serious challenge to intervention efforts in communities similar to Khayelitsha, where these perceptions strongly influence social norms. According to the theory of planned behaviour ${ }^{(39)}$, social norms, which relate to perceived social pressure, are a strong influence on an individual's intention to change his/her behaviour. Furthermore, social cognitive theory ${ }^{(40)}$ highlights the influence of the perceived behaviour of others, influenced by modelling, on behaviour change.

The barrier of community perceptions relates not only to body size and weight loss, as already mentioned, but also to weight-loss efforts in general, which would include dietary and physical activity behaviours. Participants' concerns about being stigmatised for their weight loss suggest a sense of disempowerment among participants about managing or decreasing their weight and taking steps to improve their health. They may have a positive attitude towards weight-loss efforts, the knowledge of how to change their behaviour and the sense of control over their behaviour, all believed to influence behaviour change intention according to the theory of planned behaviour $^{(39)}$, but they do not seem supported by their community to take the necessary steps towards a more healthy weight status. Feelings of disempowerment could have a negative impact on participants' sense of personal responsibility for their health, leading to a state of learned helplessness regarding their weight status and consequently their health.

Overcoming these feelings of disempowerment and helplessness may need to be included in intervention efforts in a setting where community perceptions such as those mentioned exist. Furthermore, it may be more effective in such an intervention to emphasise healthy lifestyle behaviours for the improvement of health status, rather than for weight loss. Another approach may be to focus on the prevention of weight gain, rather than weight loss. Indeed, a recent longitudinal study undertaken in free-living premenopausal women from Khayelitsha reported an average weight gain of 6.9 (SD 9.9) $\mathrm{kg}$ over a 5.5 -year period, representing an $8.8 \%$ increase in body weight $^{(41)}$. Those who were younger and had lower BMI were at greater risk for weight gain. Therefore, adopting healthier lifestyle behaviours could be more successful for the prevention of weight gain rather than weight loss. 
Other barriers to behaviour change include the community perception that healthier foods are more expensive and participants' concerns about affording healthier food if they were unemployed. This point was echoed in their comments about being willing to take part in a weight-loss intervention should they be provided with healthy foods. Indeed, lack of resources as a barrier to behaviour change is not unexpected in a community in which the average monthly household income of the majority (89\%) of households is less than R6400 (GBP 360) ${ }^{(24)}$. This highlights the challenges of implementing dietary interventions in an environment where food insecurity is a reality, but where there is a wide availability of staple foods that can be a barrier to healthy eating. Emotional overeating of these staple foods should also be addressed in such interventions. The concerning prevalence of food insecurity in urban informal areas such as Khayelitsha was one of the findings of the SA National Health and Nutrition Examination Survey ${ }^{(6)}$, and should be a consideration when developing and implementing weight-loss interventions in areas such as this.

In terms of barriers to exercise, participants highlighted the lack of facilities and equipment for exercise programmes. However, in general, participants tended to refer to exercise as intentional physical activity that would be conducted in their leisure time, with few associating it with activities of daily living, such as walking and housework. It was evident that some participants were not motivated to exercise at all. Despite negative community perceptions regarding exercise and the lack of motivation on the part of some participants, many participants showed an interest in participating in an exercise-based intervention and mentioned that there are others in the community who would be interested in participating. However, the conflicting and somewhat confusing views about who should lead such an intervention further highlight the complexities of intervening at a community level. Notably, it could be argued that these interventions should be sustainable to avoid disappointment and feelings of abandonment within community settings, which can be particularly relevant when it comes to the involvement of outside individuals or organisations.

There were certain limitations to the study. The number of focus groups was limited to five, they were not stratified by BMI and included women of a large age range (24-51 years). It is possible that the presence of older participants may have hindered younger participants' freedom to express their views. A further limitation was the small size of some of the focus groups; it is possible that the nature of the discussions and the way participants expressed themselves in the smaller focus groups could have been different from those in the larger groups. Although we aimed to include between six and eight participants per group (recruiting between eight to ten participants per focus group to allow for drop-outs), far more participants did not arrive for the focus groups than anticipated. More focus groups were scheduled to account for the low participant numbers, but the challenge persisted. Due to the deadline for completion of the data collection (the study was for an honours student project), it was not possible to continue with these efforts indefinitely.

Inclusion of more focus groups, including focus groups stratified by BMI, as well as including a mixture of normalweight and overweight/obese women, would first increase the number of participants, but also allow for greater insight into the topics discussed and could enhance the interpretation of participants' responses. However, even with a small number of focus groups including predominantly overweight and obese women $(74 \%)$, a range of complex issues were raised that highlight the need for more extensive and in-depth research. The participants in the study were selected from an urban informal setting in Cape Town and therefore represent only a small percentage of the black female population in SA. In addition, the province of the Western Cape, in which Cape Town is located, is typically viewed as more affluent than other provinces in SA. It is possible that black SA women living in more deprived settings in other SA provinces may hold different views. However, it is possible that some of the complexities around body size and weight loss may be evident among other black SA women, although the nature of these complexities may differ according to the setting.

Most of the participants in the present study were unemployed and of low socio-economic status, which was highlighted as a major barrier to behaviour change. In the future, perceptions, attitudes and knowledge of black SA women should be explored among other income groups. Areas such as Johannesburg have a greater proportion of black women of middle to high socio-economic status and a greater diversity of language (and hence cultural) groups. Future research could explore the complexities of black SA women's views from these settings, to determine the extent to which higher income and other cultural values influence these views.

\section{Conclusion}

In conclusion, body size, weight loss and weight-loss interventions are complex issues in low-income, transitioning communities and personal perceptions of these issues are strongly influenced by cultural ideals and community perceptions. The findings of the present pilot study have provided insight into these complexities, and it is essential these be considered when developing culturally appropriate weight-loss interventions that are intended to be effective in communities such as these. While it is possible that greater development and urbanisation may eventually shift social norms to facilitate a greater tolerance for weight-loss efforts, it is likely that this development and urbanisation will continue to fuel the rise of 
overweight, obesity and non-communicable diseases in transitioning communities, making the need for interventions to promote health and reduce obesity even more pressing.

\section{Acknowledgements}

Acknowledgements: The participants are thanked for their time and contribution to the research study. Nandipha Sinyanya is acknowledged for her excellent fieldwork and translation. Financial support: This research received no specific grant from any funding agency in the public, commercial or not-for-profit sectors. Conflict of interest: None. Authorship: J.H.G. and C.E.D. conceptualised and designed the study; K.J.D. was responsible for acquisition and initial analysis of the data; K.J.D., C.E.D. and J.H.G. were involved in the interpretation of the data, drafting and revision of the manuscript, and final approval of the submitted manuscript. Ethics of human subject participation: This study was conducted according to the guidelines laid down in the Declaration of Helsinki and all procedures involving human subjects/patients were approved by the Human Research Ethics Committee of the Faculty of Health Sciences of the University of Cape Town (REC REF 101/2004, Version 5, March 2012). Written informed consent was obtained from all subjects/patients.

\section{References}

1. World Health Organization (2012) Global Status Report on Noncommunicable Diseases 2010. Geneva: WHO

2. Ziraba AK, Fotso JC \& Ochako R (2009) Overweight and obesity in urban Africa: a problem of the rich or the poor? BMC Public Health 9, 465.

3. Mayosi BM, Flisher AJ, Lalloo UG et al. (2009) Health in South Africa 4. The burden of non-communicable diseases in South Africa. Lancet 374, 934-947.

4. Puoane T, Steyn K, Bradshaw D et al. (2002) Obesity in South Africa: the South African Demographic and Health Survey. Obes Res 10, 1038-1048.

5. Joubert J, Norman R, Bradshaw D et al. (2007) Estimating the burden of disease attributable to excess body weight in South Africa in 2000. S Afr Med J 97, 683-690.

6. Shisana $\mathrm{O}$, Labadarios $\mathrm{D}$, Rehle $\mathrm{T}$ et al.; SANHANES-1 Team (2013) South African National Health and Nutrition Examination Survey (SANHANES-1). Cape Town: HSRC Press.

7. Mciza Z, Goedecke JH, Steyn NP et al. (2005) Development and validation of instruments measuring body image and body weight dissatisfaction in South African mothers and their daughters. Public Health Nutr 8, 509-519.

8. Altabe M (1998) Ethnicity and body image: quantitative and qualitative analysis. Int J Eat Disord 23, 153-159.

9. Madrigal H, Sanchez-Villegas A, Martinez-Gonzalez MA et al. (2000) Underestimation of body mass index through perceived body image as compared to self-reported body mass index in the European Union. Public Health 114, 468-473.

10. Caradas AA, Lambert EV \& Charlton KE (2001) An ethnic comparison of eating attitudes and associated body image concerns in adolescent South African schoolgirls. J Hum Nutr Diet 14, 111-120.
11. Puoane T \& Mciza Z (2012) Socio-cultural and environmental factors related to obesity in black Africans: a perspective from South Africa. In Obesity: A Multidimensional Approach to Contemporary Global Issues, pp. 91-98 [R Sinha and S Kapoor, editors]. New Delhi: Dhanraj Book House.

12. Stern R, Puoane T \& Tsolekile L (2010) An exploration into the determinants of noncommunicable diseases among rural-to-urban migrants in periurban South Africa. Prev Chronic Dis 7, A131.

13. Boyington JEA, Carter-Edwards L, Piehl M et al. (2008) Cultural attitudes toward weight, diet, and physical activity among overweight African American girls. Prev Chronic Dis 5, A36.

14. Roberts A, Cash TF, Feingold A et al. (2006) Are black-white differences in females' body dissatisfaction decreasing? A meta-analytic review. J Consult Clin Psychol 74, 1121-1131.

15. Bhuiyan AR, Gustat J, Srinivasan SR et al. (2003) Differences in body shape representations among young adults from a biracial (Black-White), semirural community: the Bogalusa Heart Study. Am J Epidemiol 158, 792-797.

16. Puoane T, Tsolekile L \& Steyn N (2010) Perceptions about body image and sizes among black African girls living in Cape Town. Ethnic Dis 20, 29-34.

17. Beaglehole R \& Yach D (2003) Globalisation and the prevention and control of non-communicable disease: the neglected chronic diseases of adults. Lancet $\mathbf{3 6 2}$, 903-908.

18. Friel S, Chopra M \& Satcher D (2007) Unequal weight: equity oriented policy responses to the global obesity epidemic. BMJ 335, 1241-1243.

19. Sanders D \& Chopra M (2006) Key challenges to achieving health for all in an inequitable society: the case of South Africa. Am J Public Health 96, 73-78.

20. Matoti-Mvalo T \& Puoane T (2011) Perceptions of body size and its association with HIV/AIDS. S Afr J Clin Nutr 24, 1-6.

21. Marshall C \& Rossman GB (2010) Designing Qualitative Research. Thousand Oaks, CA: SAGE Publications Inc.

22. Wilkinson S (2004) Focus group research. In Qualitative Research: Theory, Method and Practice, 2nd ed., pp. 177-199 [D Silverman, editor]. London: SAGE Publications Ltd.

23. Patton MQ (2002) Qualitative Research and Evaluation Methods, 3rd ed. Thousand Oaks, CA: SAGE Publications Inc.

24. Statistics South Africa (2013) City of Cape Town - 2011 Census Suburb Khayelitsha. Cape Town: Statistics South Africa.

25. Garone DB, Hilderbrand K, Boulle AM et al. (2011) Khayelitsha 2001-2011: 10 years of primary care HIV and TB programmes. $S$ Afr J HIV Med 12, 33.

26. Jennings CL, Lambert EV, Collins M et al. (2009) The atypical presentation of the metabolic syndrome components in black African women: the relationship with insulin resistance and the influence of regional adipose tissue distribution. Metabolism 58, 149-157.

27. Goedecke JH, Levitt NS, Lambert EV et al. (2009) Differential effects of abdominal adipose tissue distribution on insulin sensitivity in black and white South African women. Obesity (Silver Spring) 17, 1506-1512.

28. Dickie K, Micklesfield LK, Chantler S et al. (2014) Meeting physical activity guidelines is associated with reduced risk for cardiovascular disease in black South African women; a 5.5-year follow-up study. BMC Public Health 14, 498.

29. Braun V \& Clarke V (2006) Using thematic analysis in psychology. Qual Res Psychol 3, 77-101.

30. Steyn NP, Nel JH, Parker W et al. (2012) Urbanisation and the nutrition transition: a comparison of diet and weight status of South African and Kenyan women. Scand J Public Health 40, 229-238.

31. Brewis AA, McGarvey ST, Jones J et al. (1998) Perceptions of body size in Pacific Islanders. Int J Obes Relat Metab Disord 22, 185-189. 
32. Rasheed P (1998) Perception of body weight and self-reported eating and exercise behaviour among obese and non-obese women in Saudi Arabia. Public Health 112, 409-414.

33. Shoneye C, Johnson F, Steptoe A et al. (2011) A qualitative analysis of black and white British women's attitudes to weight and weight control. J Hum Nutr Diet 24, 536-542.

34. Sivalingam SK, Ashraf J, Vallurupalli N et al. (2011) Ethnic differences in the self-recognition of obesity and obesityrelated comorbidities: a cross-sectional analysis. J Gen Intern Med 26, 616-620.

35. Blixen CE (2006) Values and beliefs about obesity and weight reduction among African American and Caucasian women. J Transcult Nurs 17, 290-297.

36. Stevens J, Kumanyika SK \& Keil JE (1994) Attitudes toward body size and dieting: differences between elderly black and white women. Am J Public Health 84, 1322-1325.
37. Christian AH, Rosamond W, White AR et al. (2007) Nineyear trends and racial and ethnic disparities in women's awareness of heart disease and stroke: an American Heart Association national study. J Women Health 16, 68-81.

38. Jefferson VW, Melkus GD \& Spollett GR (2000) Healthpromotion practices of young black women at risk for diabetes. Diabetes Educ 26, 295-302.

39. Ajzen I (1991) The theory of planned behavior. Organ Behav Hum Dec Process 50, 179-211.

40. Bandura A (1986) Social Foundations of Thought and Action: A Social Cognitive Theory. Englewood Cliffs, NJ: Prentice-Hall.

41. Chantler S, Dickie K, Micklesfield L et al. (2013) Longitudinal changes in body composition and its relation to metabolic outcomes in black South African women. J Endocrinol Metab Diabetes S Afr 18, 49. 